\title{
Models and Size Effects: A Review
}

\author{
A. Mohammed1, N. Muazu2, B. S. Waziri2 \& T. Y Ahmad1 \\ ${ }^{1}$ Lecturer, Civil Engineering Department, Abubakar Tafawa Balewa University, Bauchi, \\ ${ }^{2}$ Lecturer, Civil Engineering Department, University of Maiduguri
}

\begin{abstract}
Small scale modelling of structures is normally employed as a means to study the structural behaviour of engineering structures in order to predict the real behaviour of such structures under various conditions of loading as well as under the influence of extreme weather events like flooding, earthquakes, severe wind storms etc. But in order to use small scale models to predict prototype behaviour, understanding is necessary of the effect of scale on such models. The fracture mechanics perspective of the effects of size on models is explored from available literature and its significance to reliable model studies is established in this review.

Keywords: Models, size effects, small scale, fracture mechanics, strength.
\end{abstract}

\section{Introduction}

Historically the value in scale models lies in being able to predict the behaviour of a prototype model from the scale model. Presently small models are usually used to validate numerical models which will then be used to predict the structural behaviour of whole structures like model arch bridges and buildings. However for a researcher to be able to predict this with some degree of confidence, knowledge is required of the effect of size or scale on the model material. This becomes more important in the case of composites like masonry where the constituent brick and mortar have different properties. Therefore an appreciation is needed of the effect of size or scale on the model material, if reliable model studies are to be made.

\section{Size effect}

Scale effect is a phenomenon related to the change, usually an increase in strength that occurs when the specimen size is reduced [1]. The importance of this effect cannot be overemphasised as more and more reduced scale model studies are being undertaken for the prediction of various aspects of prototype behaviour and design strengths (that is in codes and standards). Correct understanding of size effects is also necessary for the accurate interpretation of material properties tests of various sizes and shapes in different parts of the globe.

\section{History}

The subject of size effect of objects was discussed by Leonardi da Vinci as early as the 1500's, and concluded that "among cords of equal thickness the longest is the least strong". He also added that a cord "is so much stronger ... as it is shorter"[2]. After more than a century Galileo, in 1638, rejected Leonardo's exaggerated rule and argued that cutting a long cord at various points should not necessarily make the remainder stronger. However he added that a size effect is seen because large animals have relatively bulkier bones than small ones, which he referred to as the "weakness of giants".

Some decades later Mariotte experimented with ropes, paper and tin and concluded that "a long rope and a short one always support the same weight except that in a long rope there may happen to be some faulty place in which it will break sooner than in a shorter (one)" He suggested that this is as a result of the principle of "the inequality of matter whose absolute resistance is less in one place than (in) another". In effect he had put the foundations of the statistical theory of size effect, two and half centuries before Weibull.

Griffith's [2]famous work in 1921 followed, which founded the theory of fracture mechanics and also introduced fracture mechanics into study of size effect. Griffith concluded from his observations that "the weakness of isotropic solids... is due to the presence of discontinuities or flaws. The effective strength of technical materials could be increased 10 or 20 times at least if these flaws could be eliminated". His work in effect provided an experimental basis of Mariottes's statistical concept of size effect rather than a discovery of a new type of size effect[2]. Weibull then completed the statistical size effect initiated by Mariotte in a series of papers over 17 years from 1939-1956[2]. Most of the studies thereafter until the 1980s dealt with the purely statistical origin for size effect on the strength of quasi-brittle materials, therefore until the mid 1980's size effect on strength was thought of as having a statistical origin. This is seen in Sabnis's review[3] of studies in the area in which he only discussed the known statistical concepts at that time of bundled strength and weakest link. The most notable of the statistical concepts was the latter, which holds that, the presence of a single severe defect in any of the constituent elements is adequate to cause failure of the whole material. Consequently the failure strength of a specimen subjected to uniform stress is determined by the strength of the weakest element present. The most prominent exponent of this theory was Weibull [3]. 


\section{The energetic and deterministic size effect}

In Linear Elastic Fracture Mechanics (LEFM), where the failure criterion is expressed in terms of the energy consumed per unit crack length increment. The fracture process is assumed to occur at one point, the crack tip, which exhibits the strongest possible size effect. In this case the nominal strength is inversely proportional to the square root of structure size [4]. But this is only true if the cracks are large and there is a sudden failure at the start of cracking. This theory can therefore, only be true for very large structures in which failure occurs immediately after crack initiation. However it has been observed that concrete test data do not follow LEFM criteria, neither do they obey strength theory, in which there is no size effect. Therefore there is need for a non-linear form of fracture mechanics that takes into account the stable crack growth and the large micro-cracked zone of the fracture surface. This criteria or law as shown in Figure 1, bridges the zero size effect of the strength criterion and the size effect predicted by LEFM because it recognizes the large size of the microcracked zone of quasibrittle materials like concrete, rocks and clay bricks. It is seen from the figure that most structures lie in between the strength criterion (most laboratory tests) and LEFM (very large structures like dams). This figure suggests that there may not be a strong size effect for most tests conducted in laboratories with a relatively small size for a reference structural size D (as defined below). This could apply to this investigation since the tests would be on small masonry assemblies and not large masonry structures.

The size effect derived by [5] is based on the theory of stress redistribution and fracture energy release. It is assumed that the length of a crack at maximum load is proportional to a reference structure size D (say beam depth) while the size (width) of a fracture process zone at maximum load is constant, related to the heterogeneity of the material. The fracture energy, $\mathrm{G}_{\mathrm{f}}$ is defined as a material property representing the amount of energy required to propagate a unit area of crack, then the energy used and released by fracture is proportional to $\mathrm{G}_{\mathrm{f}} \mathrm{D}[2]$. Because the energy required to produce a unit fracture extension is approximately independent of the structural size, the nominal stress at failure of a larger structure is lower than for a smaller one, so that the energy release would exactly match the energy required for the fracture formation [6].

The size effect on structural strength is taken as the effect of the characteristic structure size (say D) on the nominal strength $\sigma_{N}$ of the structure when geometrically similar structures are compared. This size effect in two dimensions is defined in terms of a nominal stress at failure in Equation 1

$\sigma_{\mathrm{N}}=\mathrm{C}_{\mathrm{n}}\left(\mathrm{P}_{\mathrm{u}} / \mathrm{bD}\right)$

Where $\mathrm{P}_{\mathrm{u}}=$ maximum (ultimate) load, $\mathrm{b}=$ thickness of specimen or structure, $\mathrm{D}=$ characteristic dimension, and $\mathrm{C}_{\mathrm{n}}=$ dimensionless constant. This is rewritten in terms of size effect of fracture mechanics type in the form of Equation 2

$\sigma_{\mathrm{N}}=\frac{B_{o} f_{t}}{\sqrt{(1+\beta)}}, \beta=\mathrm{D} / \mathrm{D}_{\mathrm{o}}$

Where $f_{t}=$ is a measure of material strength introduced to make $B_{o}$ non-dimensional and $B_{o}$ and $D_{o}$ are empirical constants; coefficients $B_{0}$ and $D_{0}$ represent specimen shape and size. This assumes that the thickness $b=$ constant for different $\mathrm{D}$ and also the specimen proportions are constant for all sizes. When the specimen is small, plasticity is also small, size effect does not manifest at these smaller values of $\beta$, and effectively results in a horizontal curve as seen in Figure 1. At intermediate values, there is a smooth transition and in the case of LEFM at large sizes $(\beta \rightarrow \infty)$ at which case size effect is very pronounced it approaches the asymptote with slope of $1: 2[1]$.

Equation 2 can be transformed to a linear plot in the form of Equations 3 and 4 $\mathrm{Y}=\mathrm{AX}+\mathrm{C}$

Where $\mathrm{X}=\mathrm{D}, \mathrm{Y}=\frac{1}{\left(\sigma_{N}\right)^{2}}, \mathrm{~A}=\frac{1}{D_{o} B_{o}{ }^{2} f_{t}^{2}}$, and $\mathrm{C}=\mathrm{AD}_{\mathrm{o}}$

The intercept $\mathrm{C}$ and slope $\mathrm{A}$ can be determined through linear regression analysis of data.

Some criticisms abound in the literature regarding the applicability of the law to unnotched structures. [7] observed that in deriving the formula the energy dissipated to cause fracture in a notched specimen is proportional to $G_{f} D$, and $D$ is proportional to the crack length. But for unnotched specimens the length of the characteristic flaw responsible for crack propagation is independent of specimen size, which therefore causes the anomalous behaviour of Bazant's formula. However [8] argues it is misleading to use Equation (1) for unnotched specimens since its modification has been derived to cater for unnotched specimens as given in Equation 5; 
$\sigma_{N}=k \sigma_{0}\left(1+\frac{r D_{b}}{D}\right)^{1 / r}$

Equation (5) was derived for the modulus of rupture of a notchless concrete beam, where $\sigma_{0}=(2 D / 3 L) f_{t}$ and $D_{b}$ is the thickness of the boundary layer of cracking are both constants because the ratio $\mathrm{D} / \mathrm{L}$ is constant for geometrically similar structures. $\mathrm{D}$ and $\mathrm{L}$ are the beam depth and length respectively, $\mathrm{r}$ and $\mathrm{k}$ are positive constants (usually $\mathrm{k}=1$ and $\mathrm{r}=1$ or 2)[9].

The theory of Crack Fractality or the Multifractal Scaling Laws (MFSL).

Theory of crack fractality or the Multifractal scaling Laws (MFSL) can be of two types;

1. Invasive fractality of the crack surface; referring to the fractal nature of surface roughness.

2. Lacunar fractality; referring to the fractal distribution of microcracks.

This theory proposed by Carpenteri and his co workers[10-12] holds that the difference in fractal characteristics of cracks at different scales of observation is the main source of size effect in disordered materials. The nominal stress from this law is given by Equation 6

$\sigma_{U}=f_{t}\left(1+\frac{l_{c h}}{b}\right)^{1 / 2}$

Where $b$ is the characteristic dimension of the structure and $l_{\mathrm{ch}}$ is a characteristic length related to the material microstructure. The scaling relationship shown in Figure 2(a) is a two-parameter model, where the asymptotical value of the nominal strength $f_{t}$ corresponding to the lowest tensile strength is reached only in the limit of infinite sizes. The dimensionless term $\left(1+1_{\mathrm{ch}} / \mathrm{b}\right)$ in Equation 6 represents the variable influence of disorder, consequently quantifying the difference between the nominal quantity measured at scale $b$ and the asymptotic value. In the logarithmic plot shown in Figure 2(b), the transition from the fractal regime to the homogeneous becomes clear. The threshold of this transition is represented by point $\mathrm{Q}$, whose ordinate and abscissa are $\log \mathrm{f}_{\mathrm{t}}$ and $\log \mathrm{l}_{\mathrm{ch}}$ respectively.

Some observations have been made by $[2,8]$ on the MFSL some of which are:

1. That the MFSL is identical to a special case of the energetic- statistical scaling law for failure at crack initiation (Equation (5)).

2. That the derivation of MFSL from fractal concepts includes problematic steps which invalidate it and that the MFSL does not follow mathematically from the fractal hypothesis made by its proponents.

3. That the MFSL cannot predict the dependence of size effect law parameters on the structure geometry. On the other hand, the energetic theories are able to predict their dependence.

It can be seen that taking $\mathrm{k}=1$ and $\mathrm{r}=2$ in Equation (5) gives

$\sigma_{N}=\sigma_{0}\left(1+\frac{2 D_{b}}{D}\right)^{1 / 2}$

The remarkable similarity between Equations 5 and 7 is then apparent, and the constants in the equations can easily be obtained through statistical regression of test data as before.

\section{Karihaloo's size effect formula for notched quasi-brittle structures}

[13] used the concept of a fictitious crack model (FCM) to arrive at a size effect formula for the nominal strength of notched three point bend fracture specimens of concrete and other quasi-brittle structures defined as

$\sigma_{N}=A\left(1-\frac{B}{W}\right)^{1 / 2}$

Where $A=\sigma_{\infty} ; \quad B=\frac{1}{2} l_{p \infty} \frac{g^{\prime}(\alpha)}{g(\alpha)}$

Where $l_{p \infty}$ and $\sigma_{\infty}$ refer to the size of the fracture process zone in a very large specimen $(W \rightarrow \infty)$ and its nominal strength, while $g(\alpha)$ and $g^{\prime}(\alpha)$ are a non- dimensional geometry factor and its first derivative, 
respectively. They depend on the notch to depth ratio $\alpha=a / W$. Deficiencies and refinements to Equation (8) have been discussed by [14] and [15] and will not be covered here.

\section{Factors affecting size/scale Effect}

A number of factors affect the strength properties and ultimately the overall behaviour of quasi-brittle materials. Some of the strength properties include compressive, tensile, tensile, bond and fatigue strengths, and various dimensional changes including creep. The materials affected by size effect range from naturally occurring rock, timber etc to man made materials like concrete, steel etc and even composites like masonry[16].

\section{Random strength}

Random strength describes the effect random distribution of flaws has in a heterogeneous material. It plays an important role in the micro mechanisms determining the strength of materials. Thus a larger specimen should have more flaws and consequently less strong than a smaller specimen with fewer flaws[1].

\section{Compaction}

Generally smaller specimens of mortar or concrete will tend to achieve better compaction and higher density and thus a higher strength because of their smaller volumes. This is especially the case when standard compaction procedures are employed through vibration for a given time or specific number of tampings[1].

\section{Curing and drying}

Curing is an important variable influencing mortar strength. Two specimens of different sizes will cure differently because the surface to volume ratio increases with decrease in specimen size. The strength of the material will vary from the surface of the specimen to its centre, depending on its size, since hydration may not be uniform throughout the specimen at the time of testing[1].

Drying of the specimen will also influence the gain in strength as a result of the surface to volume ratio, which varies inversely with the specimen size[1].

\section{Loading rate and state of stress}

The rate of loading influences the strength of specimens, because higher loading rates lead to higher strengths. The stress of stress, for example compression, tension, and flexure, also influences the strength of the specimen. For instance the strength of compressive specimens depends on the accuracy of the loaded ends, and on parallelism, if rotating heads are not used. It is also possible to achieve a higher level of capping accuracy in smaller specimens, which will result in higher strength[1].

\section{Testing machine and loading platens}

Properties of the testing machine such as the stiffness of the loading platens have a profound effect on test results. Stiff end plates tend to apply uniform strain conditions to the specimen under test, thus resulting in a higher strength than if thinner plates were used[1].

\section{Compaction of mortar bed by masonry units}

Because of the heavier masses of larger masonry units, they tend apply more pressure on the mortar bed than smaller units. This observation was made by [17] as a possible cause for the decrease in stiffness as masonry model size is reduced.

\section{Conclusions}

The forgoing has shown the importance of size effects if reliable and repeatable model studies are to be made. The importance of this effect cannot be overemphasised more so, because of the different specimen sizes around the world and also as more and more reduced scale model studies are being undertaken for the prediction of various aspects of prototype behaviour and design strengths in codes and standards. 


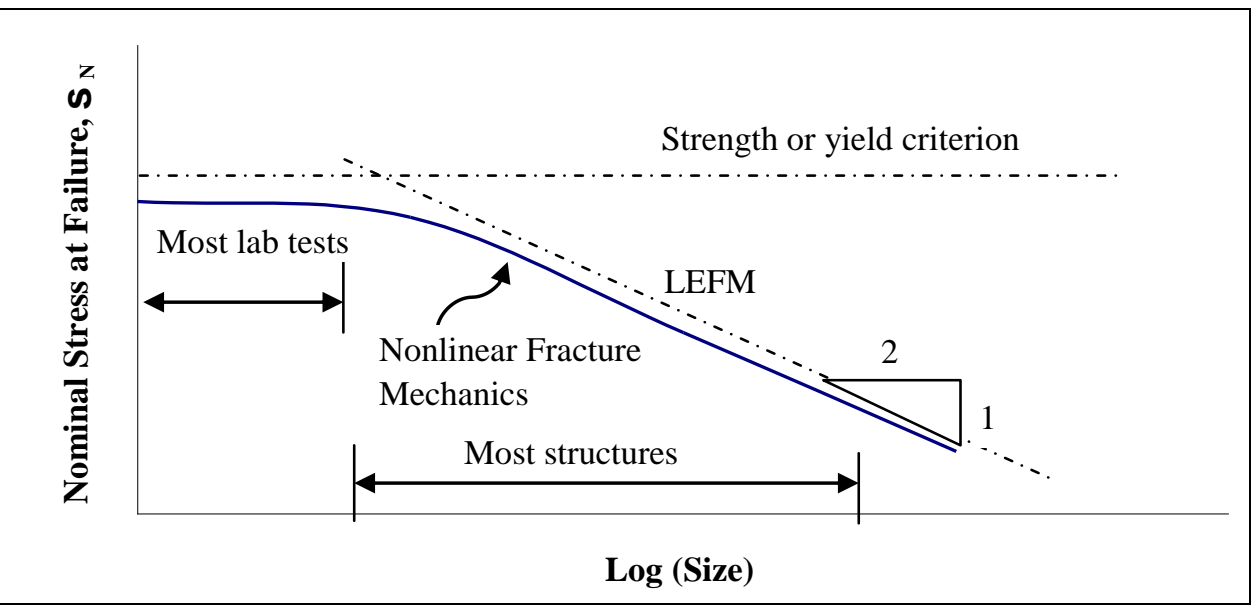

Figure 1- Size effect according to strength criteria, linear and nonlinear fracture mechanics.
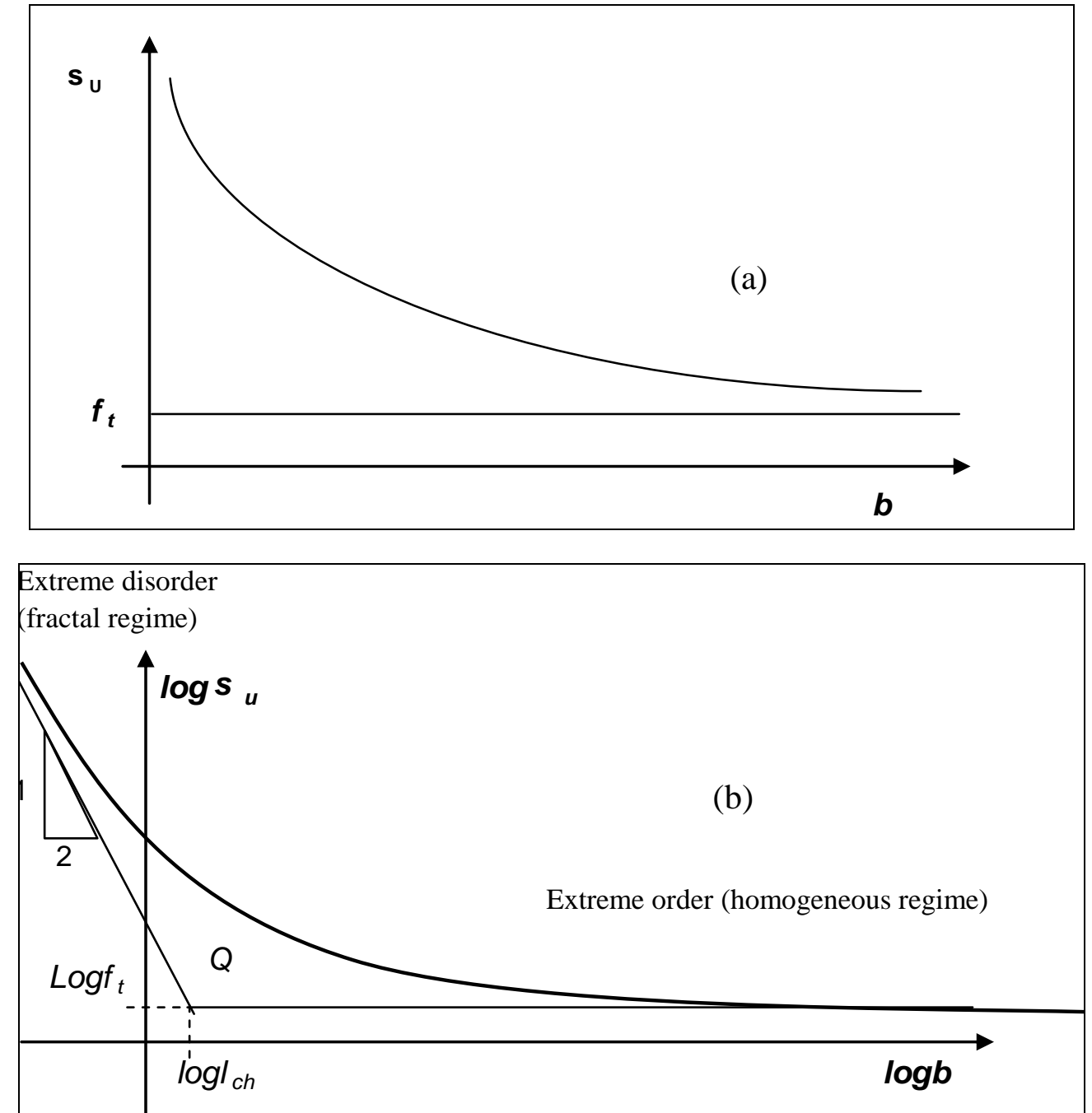

Figure 2 - (a) Multifractal scaling law (MFSL) for tensile strength, (b) Bilogarithmic diagram for the same.

\section{References}

[1]. Harris GH, Sabnis GM. Structural Modelling and Experimental Techniques. 2nd ed. Florida: CRC Press LLC 1999.

[2]. Bazant ZP. Scaling of structural strength. London: Hermes Penton Ltd 2002

[3]. Sabnis GM. Size effects in material systems and their impact on model studies: A theoritical approach. Proceedings of SECTAM X conference. Knoxville, TN 1980:649-68.

[4]. Bazant ZP. Size effect. International Journal of Solids and Structures. 2000;37:69-80.

[5]. Bazant ZP. Size effect in blunt fracture: concrete, rock, metal. Journal of Engineering Mechanics, ASCE. 1984;110:518-35 
[6]. Lourenco PB. Two aspects related to the analysis of masonry structures: size effect and parameter sensitivity.: Delft University of Technology Faculty of Civil Engineering 1997. Report No.: 97-NM-R1533.

[7]. Carpenteri A, Chiaia B. Embrittlement and decrease of apparent strength in large-sized concrete structures. Sadhana. 2002;Part 4(27):425-48.

[8]. Bazant Z, Yavari A. Is the cause of size effect on structural strength fractal or energetic-statistical.? Engineering fracture mechanics. 2005(72):1-.31.

[9]. RILEM. RILEM TC QFS - Quasibrittle frcature scaling and size effect- Final report. Materials and Structures. 2004 October 2004;37:547-68.

[10]. Carpenteri A, Chiaia B. Multifractal scaling law for the fracture energy variation of concrete structures. In: Wittmann FH, editor. Proceedings FRAMCOS-2; 1995; Freiburg, Germany: Aedificatio Publishers; 1995. p. 581-96.

[11]. Carpenteri A. Fractal nature of material microstructure and size effects on apparent mechanical properties. Mechanics of materials. 1994(18):89-101.

[12]. Carpenteri A, Ferro G. Size effects on tensile fracture properties: a unified explanation based on disorder and fractality of concrete microstructure. Materails and Structures. 1994(27):563-71.

[13]. Karihaloo BL. Size effect in shallow and deep notched quasi-brtittle structures. International Journal of fracture. 1999;95:379-90.

[14]. Abdalla HM. Strength size effect in concrete structures. Cardiff: Cardiff University; 2003.

[15]. Karihaloo BL, Abdalla HM, Xiao QZ. Size effect in concrete beams. Engineering fracture mechanics. 2003;70:979-93.

[16]. Sabnis GM, Aroni S. Size effect in Material systems- The state of the art. Proceedings of the Southampton 1969 Civil Engineering Materials Conference. Southamptom 1971:131-42.

[17]. Egermann R, Cook DA, Anzani A. An investigation into the behaviour of scale model brick walls. Proceedings of the 9th International Brick/Block Masonry Conference; 1991; Berlin; 1991. p. 628-35. 